\title{
FORMULATION OF QUANTUM MECHANICS ON POINCARÉ DISKS
}

\author{
Arturo Tozzi \\ Center for Nonlinear Science, Department of Physics, University of North Texas, Denton, Texas, USA \\ 1155 Union Circle, \#311427Denton, TX 76203-5017 USA
}

ASL Napoli 2 Nord, Distretto 45, Caivano, Naples, Italy

Via Santa Chiara, 80023, Caivano, Naples, Italy

tozziarturo@libero.it

arturo.tozzi@alsnapoli2nord.it

\begin{abstract}
The unexploited unification of general relativity and quantum mechanics (QM) prevents the proper understanding of the micro- and macroscopic world. Here we put forward a mathematical approach that introduces the problem in terms of negative curvature manifolds. We suggest that the oscillatory dynamics described by wave functions might take place on hyperbolic continuous manifolds, standing for the counterpart of QM's Hilbert spaces. We describe how the tenets of QM, such as the observable A, the autostates $\psi$ a and the Schrodinger equation for the temporal evolution of states, might work very well on a Poincaré disk equipped with rotational groups. This curvature-based approach to QM, combined with the noncommutativity formulated in the language of gyrovectors, leads to a mathematical framework that might be useful in the investigation of relativity/QM relationships. Furthermore, we introduce a topological theorem, termed the punctured balloon theorem (PBT), which states that an orientable genus-1 surface cannot encompass disjoint points. PBT suggests that hyperbolic QM manifolds must be of genus_ $\geq 1$ before measuring and genus zero after measuring. We discuss the implications of PBT in gauge theories and in the physics of the black holes.
\end{abstract}

KEYWORDS: Einstein; manifold; Hilbert space; observer; measurement; matrix.

General relativity (GR) and quantum mechanics (QM) describe the nature through mathematical tools such as tensors and probability theory (Yilmaz, 1982; Comte, 1996; Fre, 2013). GR and QM display very different behaviors and features, starting from the subtending mathematical manifolds. General relativity entails a 3+1-dimensional pseudo-Riemannian manifold with tensor fields, while quantum field theory entails a $\mathrm{R}^{4}$ projective Hilbert space with operator-valued fields (Tegmark 2008). In the Euclidean framework we attain vectors equipped with just one basis, while in the projective infinite-dimensional Hilbert space of QM we attain vectors with different bases standing for sets of equivalence classes. On one hand, the macroscopic world of GR is equipped with an orthonormal basis common to all the observables, i.e., a set of vectors in Euclidean or Hilbert spaces such that all vectors have length one and any two of them are orthogonal and every vector can be written as a linear combination of vectors (Tumulka, 2009). On the other hand, the microscopic world of QM displays countless bases that are the source of the counterintuitive physical effects experienced during experimental observation.

Despite GR and QM work very well in the description of different features of Nature, they are seemingly not comparable and not reducible to a self-consistent unifying framework. The numerous efforts provided to correlate GR and QM paint a somewhat incomplete picture (Ghose, 1997; Skalsky, 2010; Fre, 2013; Elitzur, Dolev, Kolenda, 2017; Aerts, 2017). We provide a few examples and their limitations. Piazza et al. (2015) pointed towards the possibility of simultaneous observation of quantization and interference patterns. Nonetheless, their approach stands for a technical device rather than a proper conceptual framework able to unify GR and QM. Other investigations aimed to provide transformation rules between GR and QM manifolds: e.g., Feffman et al. (2015) assessed the geometric Whitney problem on how a Riemannian manifold can be constructed to approximate a metric space. Deformation quantization such as the WignerWeyl transform permits the achievement of a complete phase space formulation of QM equivalent to the Hilbert-space operator representation, with star-multiplications standing for isomorphically parallel operator multiplications (Curtright et al., 2014). By expressing QM in the same phase space of classical mechanics, the achieved map recognizes QM as a deformation of the classical one. However, these approaches limit themselves to the description of mere representation changes from Hilbert space to phase spaces, since they cannot generate a successful quantization scheme, namely a method to produce a quantum theory out of a classical theory.

Here we suggest a different approach towards the unification of GR and QM. Starting from the evaluation of quantum oscillations on a Poincaré disk, we examine the possibility that the oscillatory dynamics described by quantum mechanics take place on microscopic negative-curvature, hyperbolic continuous manifolds. Further, we introduce a novel topological theorem suggesting that the genus of the QM manifold modifies before and after experimental quantum measuring. 


\section{HYPERBOLIC DYNAMICS AS A CORNERSTONE OF QUANTUM MECHANICS}

Dynamical features of QM can be described in terms of wave movements on hyperbolic manifolds since it is feasible to map the Hilbert space to a negative-curved phase space. In the sequel, we define the counterparts of QM postulates in terms of the concave features of geodesic flows. Geodesic flows have the unvaluable advantages to display well-known, manageable and easily quantifiable topological properties, ergodic features, frame/horocycle flows, and so on (Pesin, 1981). Here we focus on the relatively straightforward Poincare ball model, which stands for the n-dimensional hyperbolic space in the $n$-dimensional unit ball in $\mathrm{R}_{\mathrm{n}}$. We favour the Poincaré ball model instead of other hyperbolic models such as, e.g., the Beltrami-Klein model, because the former is conformal, so that its angles/circles are preserved and easier to assess.

Before measuring. Every quantum physical system is described by a (topologically) separable complex Hilbert space of states $\mathrm{H}$ with inner product in which each vector can be decomposed in the linear combination of other vectors (Cassinelli and Lahti, 2017). Every observable A in the space $\mathrm{H}$ is associated with an hermitian linear self-adjoint operator and with an orthonormal basis of vectors, i.e., the base of its autostates wa (Dirac 1925; Gisin and Fröwis, 2018). The autovalue a of the operator A displays one or more autostates $\psi$ a. Since each autovalue a depicts a possible value of A, $\psi$ a forms a compete set, i.e., a wave function, standing for the superposition of all the $\psi$ a. Before the measurement of the observable A, all the autostates $\psi$ a are superimposed, giving rise to a wave function which completely describes the state of the system:

$\mathrm{A} \psi \mathrm{a}=\mathrm{a} \psi \mathrm{a}$

In search of relationships between the dynamics of quantum oscillations and the movements on a hyperbolic manifold, we suggest that the features of the Hilbert space, the observable A and the autostates wa before and after measuring could be described in terms of their negative-curvature counterparts on the Poincaré disk. Since the spectrum of an operator corresponds to the set of its autovalues that can be discrete or continuous, the continuous manifold of the Poincaré disk is split in tessellated areas, giving rise to countless discrete tiles. The physical pattern occurring before the experimental measurement can be illustrated in terms of the virtually uncountable number of $\psi$ a, each one filling a single tile of the Poincaré disk (Figure 1A). By the standpoint of the observer, the most of autostates $\psi$ a are difficult to access, since the most of them are in the periphery of the Poincaré sphere. In turn, by the standpoint of the observer, it is much easier to assess the largest tiles in the center of the Poincaré disk. We shall see in the sequel that this observation has considerable implications after the experimental measurement. The QM wavefunction evolves in time according to the time-dependent Schrödinger equation (Batygin, 2018). In touch with this postulate, one of the most important features of our QM hyperbolic model is that the continuous QM Poincaré disk is not at all a static manifold, rather it is equipped with rotations that provide the tiles with effortless physical movements. This permits the shifts of the tessellated areas from the center to the periphery of the disk surface, and vice versa (Figure 1B). Once described the dynamical tiles detectable by the observer before measuring, we will describe what happens to the tiles after the measurement.

After measuring. After the experimental measurement of the observable A, the system collapses towards one of its $\psi \mathrm{a}$ autostates, chosen based on probability. Every $\psi$ a corresponds to a physical state in which, after the measurement of A, one finds one of the values $\mathrm{a}_{\mathrm{n}}$ or $\mathrm{a}_{(\mathrm{f})}$, so that just a single autostate $\psi$ a becomes detectable (Figure 1C). We suggest that the very operation of measurement selects a single tile located inside the rotating Poicaré disk. Incidentally, the movements must be random, because QM, according to another of its postulates, is described by statistical results of continuous probability, where the square module of the vector coefficients stand for probabilities. Before the measurement, the tiles are free to move on the surface of the Poincare disk, following hyperbolic paths. In turn, the experimental measurement "freezes" the movements of the tessellated areas so that, after the experiment, just a single motionless tile can be detected by the standpoint of the observer. The experimental procedure of measurement generates a stationary window of observation located at the center of the Poincaré disk, where the largest tile stands for the tessellation which is the most accessible to the observer. To provide an analogy, think to the inspection of a single "frozen" frame extracted from a long video sequence. Paraphrasing Cartas-Fuentevilla et al. (2020), pathologies associated with the possible existence of many unitarity inequivalent representations on the hyperbolic disk disappear, since the whole operation of QM measurement is realized by choosing only one representation, i.e., the $\psi$ a located in the central tile.

Matrices and noncommutativity in QM. In QM, the operator is linear and can be represented as a matrix. The Taylor series development grants the hurdle of operator functions to be reduced to operations of sum and multiplication among matrices. In a base of autovectors, the operator is represented by a diagonal matrix. The simplest solution, i.e., the base of common autovectors, can be uncovered just in case the operators commute. To every observable in classical mechanics, there corresponds a linear, Hermitian operator in quantum mechanics. Instead of using matrices, in concave QM models we can use gyrovectors, i.e., the hyperbolic vectors introduced by Ungar (2005). A gyrovector space approach to analytic hyperbolic geometry is analogous to the vector space approach to Euclidean geometry. In touch with our definition of autostates, the sides of a hyperbolic geometric figure are uniquely determined by its hyperbolic 
angles, unlike Euclidean geometric figures. Further, a relationship has been demonstrated between Einstein (Möbius) gyrovector spaces and Beltrami-Klein (Poincaré) ball models of hyperbolic geometry (2005). It is noteworthy that the special grouplike loops termed gyrocommutative gyrogroups are compatible also with the special theory of relativity, in particular with the nonassociativity of Einstein's velocity addition law (Ungar, 2013).

Talking about operations of sum and multiplication among matrices, it is noteworthy that a further notable difference does exist between the two physical frameworks of GR and QM, i.e., the mathematical operations of commutativity and noncommutativity (Goodman, 2003). In the macroscopic world dictated by GR, the binary operation of multiplication is usually commutative, i.e., changing the order of the operands does not change the result. This means that: $a b=b a$. On the contrary, in QM the linear operators representing a pair of physical variables do not commute, rather they are mutually complementary. This means that $\mathrm{ab} \neq \mathrm{ba}$. Examples of complementary quantum properties that cannot be simultaneously observed/measured are position and momentum, energy and duration, spins on different axes, wave and particle, entanglement and coherence (Kalckar et al., 1996).

Efforts have been provided to tackle special relativity by the noncommutative standpoint too (Besnard and Bizi, 2017). Girelli and Livine (2004) argued that Deformed Special Relativity is obtained by imposing a maximal energy to special relativity and by deforming the Poincaré symmetry to accommodate this requirement. Variations of this procedure lead to noncommutative space structures that preserve conservation laws. In turn, the Very Special Relativity's (VSR) approach suggests that the subgroup of the Poincare group is enough for the description of the spacetime symmetries of observed physical phenomena. Das et al. (2011) introduced a novel noncommutative spacetime structure enjoying the symmetries of deformed VSR. This tricky procedure allowed them to build a point particle Lagrangian that lives in a noncommutative phase space. Some Authors view QM as a deformation of the combinatorial or Hamiltonian quantization of three-dimensional gravity in the Chern-Simons formulation (Schroers, 2011). In this case, quantum groups replace the local isometry groups and noncommutative spacetimes replace the classical model spacetimes. Kalau and Malze (1995) derived an action for gravity in the framework of noncommutative geometry by using the Wodzicki residue. They achieved a gravity action for commutative geometry which is the usual Einstein-Hilbert action. Also, they provided a noncommutative extension given by the tensor product of the algebra of smooth functions on a manifold and a finite dimensional matrix algebra. By assessing inflation on a noncommutative space-time within the framework of enveloping algebra approach, Calmet and Fritz (2015) showed that the dominant effect of the noncommutativity of spacetime comes from contributions to the process of structure formation. The last, but not the least, an extension of Connes' notion of even spectral triple to the Lorentzian setting has been proposed (Connes, 1994; Besnard and Bizi, 2017). It turns out that the data available in noncommutative geometry (i.e., the algebra of functions, the Krein space of spinor fields, the representation of the algebra on it, the Dirac operator, charge conjugation and chirality) play a role in this characterization. In this approach towards a noncommutative generalization of the modern theories of spacetime and matter, only space and time-oriented spin manifolds of even dimension are considered.

It is noteworthy that the pairs $(1,1)$ in a linear matrix won't work with hyperbolic grids. To provide an example Figure 2 illustrates that the two tiles UR and RU in the Poincaré disk cannot commute. Furthermore, the transformation matrix in Euclidean spaces that gives rise to combined matrix is transformed in combined gyrovectors in hyperbolic spaces. This means that noncommutativity can be expressed in the language of gyrovectors, such that, for example,

$(A \oplus B) \oplus C \neq A \oplus(B \oplus C)$

In sum, the Poincaré disk displays many of the features of QM, including noncommutativity. 
A
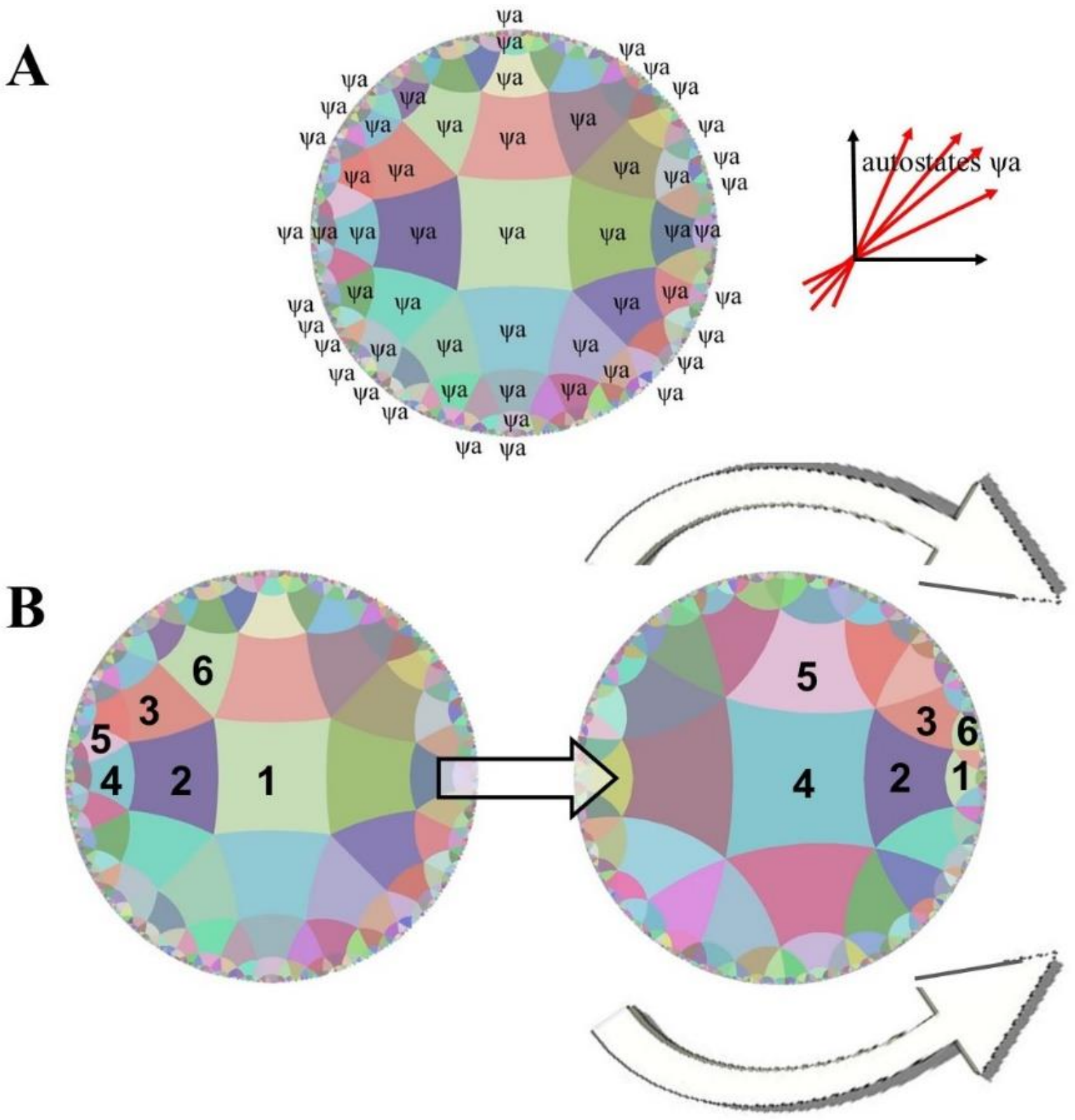

C
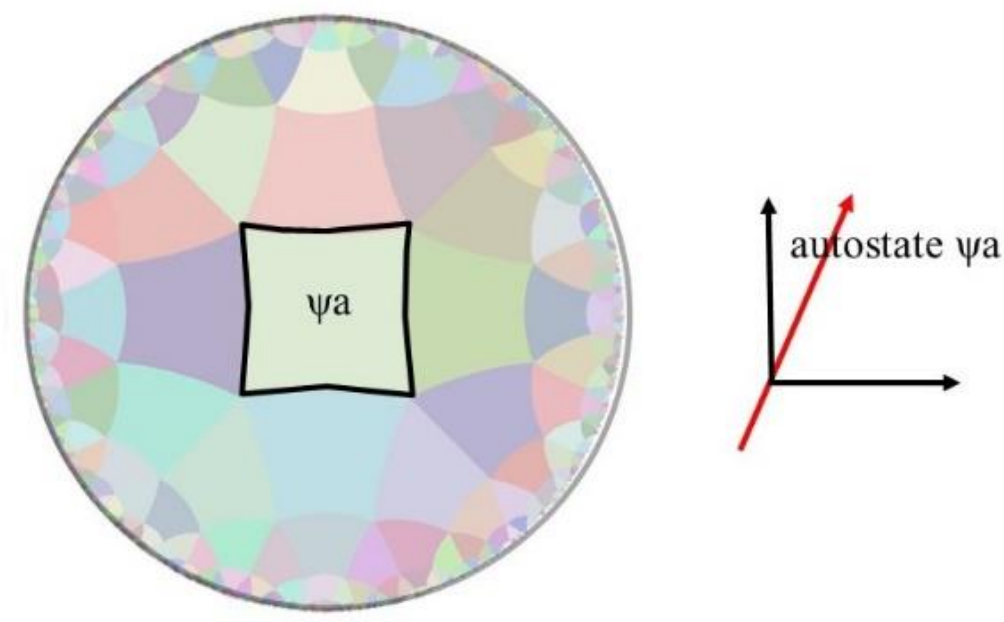

Figure 1. An observable of a QM system is portrayed in terms of a collection of tiles on a hyperbolic Poincaré disk. The Poincaré disk's tiles are painted in different colors to emphasize that each tessellation corresponds to a single autostate wa. Figure 1A. Before the experimental measurement of the observable A, all the autostates $\psi$ a are detectable, since no preferential autostate can be chosen. Before measuring, the wave function of the autostates $\psi$ a is described by a sheaf of 
vectors lying on orthonormal basis (right Figure). Figure 1B. The operations occurring on the Poincaré disk permit the movement of every tile. For sake of clarity, some of the tiles are numbered to show their movements from the initial state (left) to the final state (right). In long times, every QM wave subject to action necessarily crosses the central tile of the Poincaré disk: to provide an example, the peripheral tile 4 in the left Figure moves towards the center of the disk in the right Figure. Figure 1C. After the experimental measurement of the observable A, the system is "frozen", i.e., it collapses so that just a single autostate $\psi$ a becomes detectable. After measuring, the wave function of the autostates $\psi$ a is described by a single vector lying on orthonormal basis (right Figure). Once the QM system has been "immobilized" by the measurement, the probabilistically selected single autostate is detectable in the central tile, in case we have chosen this central tile as the frame of our observation. Modified from: Rendering Hyperbolic Spaces - Hyperbolica Devlog \#3 (https://www.youtube.com/watch?v=pXWRYpdYc7Q)

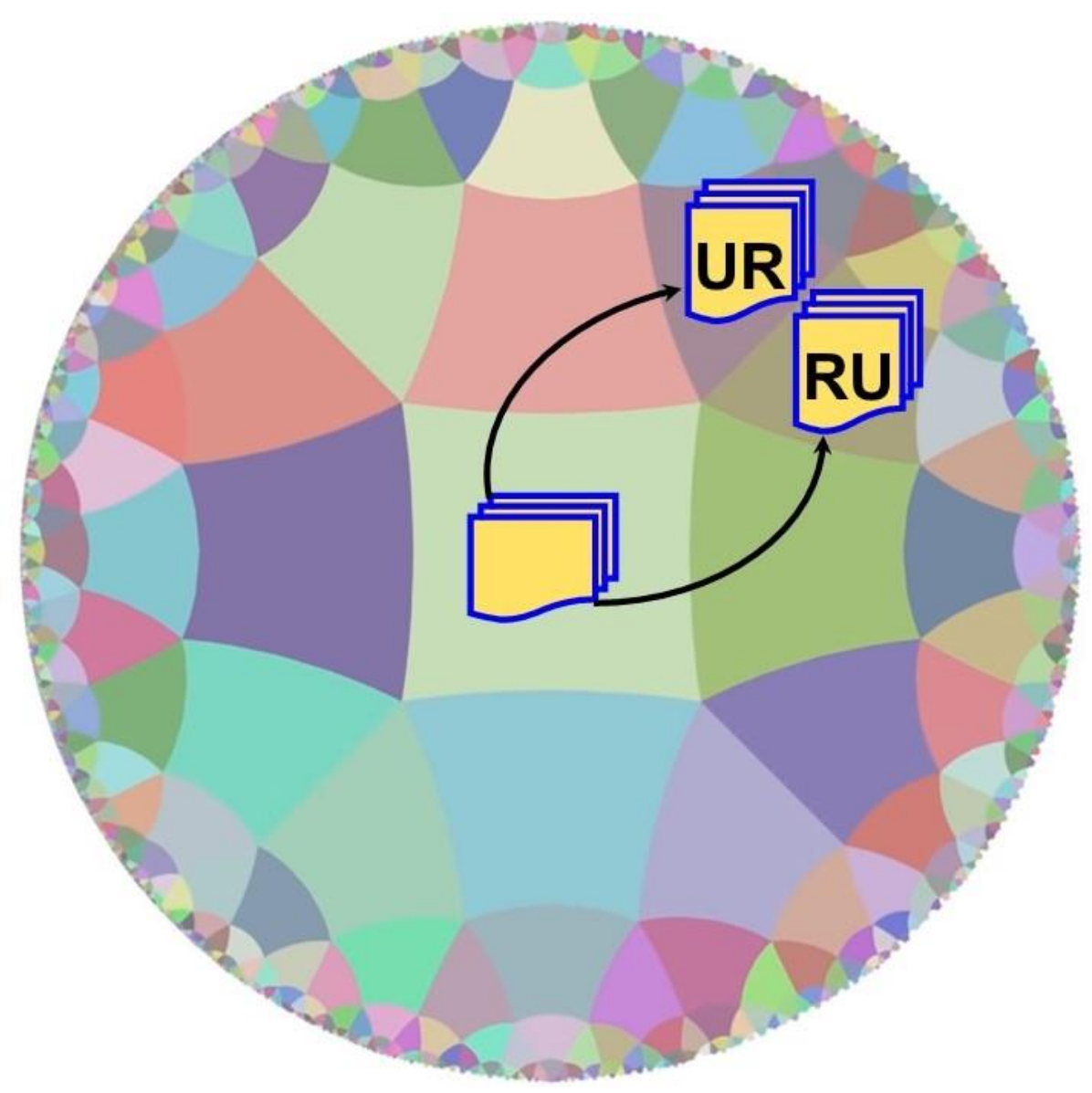

Figure 2. Noncommutative features of a Poincaré disk. The movements on a Poincaré disk are not commutative: for example, a mapping from the central tile to the tile Up Right (UR) does not commute with a mapping from the central tile to the tile Right Up (RU). 


\section{LACK OF DISJOINTNESS IN GENUS-1 SURFACES: THE PUNCTURED BALLOON THEOREM}

Two sets are disjoint if they have no elements in common, or in other words, if their intersection is an empty set (Cormen et al., 2001). Many topological manifolds contain disjoint subsets such that $\mathrm{a} \cap \mathrm{b}=\varnothing$. To provide an example, the opposite antipodal points on the genus- $0 \mathrm{n}$-sphere described by the Borsuk-Ulam theorem cannot meet, therefore are disjoint (Matoušek 2003; Peters 2016).

Here we provide a novel theorem, colloquially labelled the punctured balloon theorem (PBT), confronting jointness and disjointness of different genus manifolds.

\section{Theorem}

- An orientable genus-1 surface cannot encompass disjoint points. See the upper Figure 3 for a pictorial rendering.

\section{Proof}

- Let us assume that a genus-1 surface contains two (or more) disjoint points, such that a $\cap \mathrm{b}=\varnothing$ (lower Figures 3).

- When a deformation retraction is performed, it unavoidably happens that two (or more) disjoint points a and $\mathrm{b}$ (lower left Figure 3):

a) Approach the hole border.

b) End up closer.

- Because the two (or more) disjoint points a and b cannot meet, they leave an empty region connecting the internal of the hole with the external of the genus-1 surface.

- This would mean that the hole gets lost, being the manifold of genus-0 instead of the genus- 1 advocated in the assumption (lower left Figure 3).

- This is a contradiction; hence the assumption must be incorrect.

In plain terms, take a balloon, that is a genus-one manifold. If you break the jointness by piercing its surface, the hole gest lost and the punctured balloon becomes a genus-0 manifold. Starting from this trivial claim, we proved a topological theorem which plainly states that "the ends of a donut can meet, whilst the ends of a kidney pie cannot". Therefore, a manifold including two (or more) disjoint points turns out to be a genus-0 manifold. Note that we did not use the term "connected" in our definition of surface, since PBT casts doubts on the role of topological connectedness in the assessment of both sets and subsets. Even though deformation retraction stands for a mapping that captures the idea of continuously shrinking a space into a subspace, PBT suggests that a genus-0 manifold must necessarily encompass (at least two) nonintersecting elements.

PBT rules out the occurrence of continuity/indivisibility among the elements of a physical genus- 0 manifold, holding instead to a discrete operational approach. In touch with this observation, when a choice is performed, e.g., a gauge is introduced in a symmetric manifold (Sengupta et al., 2016), disjoint subsets are generated and manifold connectedness gets lost. This means that a genus-0 manifold, although theoretically shrinkable, must contain some disconnected elements. Take the Poincaré-Brouwer theorem, also termed the "hairy ball" theorem (HBT), stating that there is no nonvanishing continuous tangent vector field on even-dimensional $n$-spheres (Eisenberg and Guy, 1979). This implies that the poles of a genus- 0 sphere are disjoint from the neighboring elements. PBT suggests that the vanishing tangent vector fields disappear when the ball is drilled completely through: in this case, since the poles have been removed, we achieve a genus-one torus instead of a ball and HBT does not hold anymore.

PBT implies that a genus-1 surface contains just intersecting, neighborhood points. When shrinking a genus-1 space into a subspace, BPT entails that all the points gather, apart from the ones inside the hole. Therefore, a genus- 1 manifold, despite being a set that is not simply connected, must encompass just connected elements, except for the region occupied by the hole.

PBT affects not only disjoint points, but also disjoint vertexes, shapes, functions, vectors, energies, and so on (for a survey, see Tozzi et al., 2017). In physical terms, PBT suggests that a genus-1 manifold must contain homogeneous elements/features/functions all around the hole, since every part of a genus-1 system is required to topologically intersect with every other part. Therefore, in a physical system encompassing holes - i.e., vortices/antivortices (Padavić et al., 2020), Betti number $\beta_{1} \geq 1$ (Don et al., 2020), topological defects in nanoscopic materials, fragile topology features (Po et al., 2018) and so on - all the regions surrounding the impurities must display at least a few homogeneous and ergodic elements/features/functions.

Concerning the hyperbolic counterparts of QM postulates, PBT suggests that a homogeneous Poincaré disk must encompass a manifold of genus $\geq 1$ before quantum measuring. Indeed, the autostates $\psi$ a before measurement are not 
discernible on a Poincaré disk, therefore they are arbitrarily near to each other and do not contain disjointed elements. In turn, the QM manifold becomes genus zero after the experimental measurement. The measurement corresponds to the loss of genus-one (or higher) in the QM manifold, so that a single $\psi$ a becomes assessable after measuring.

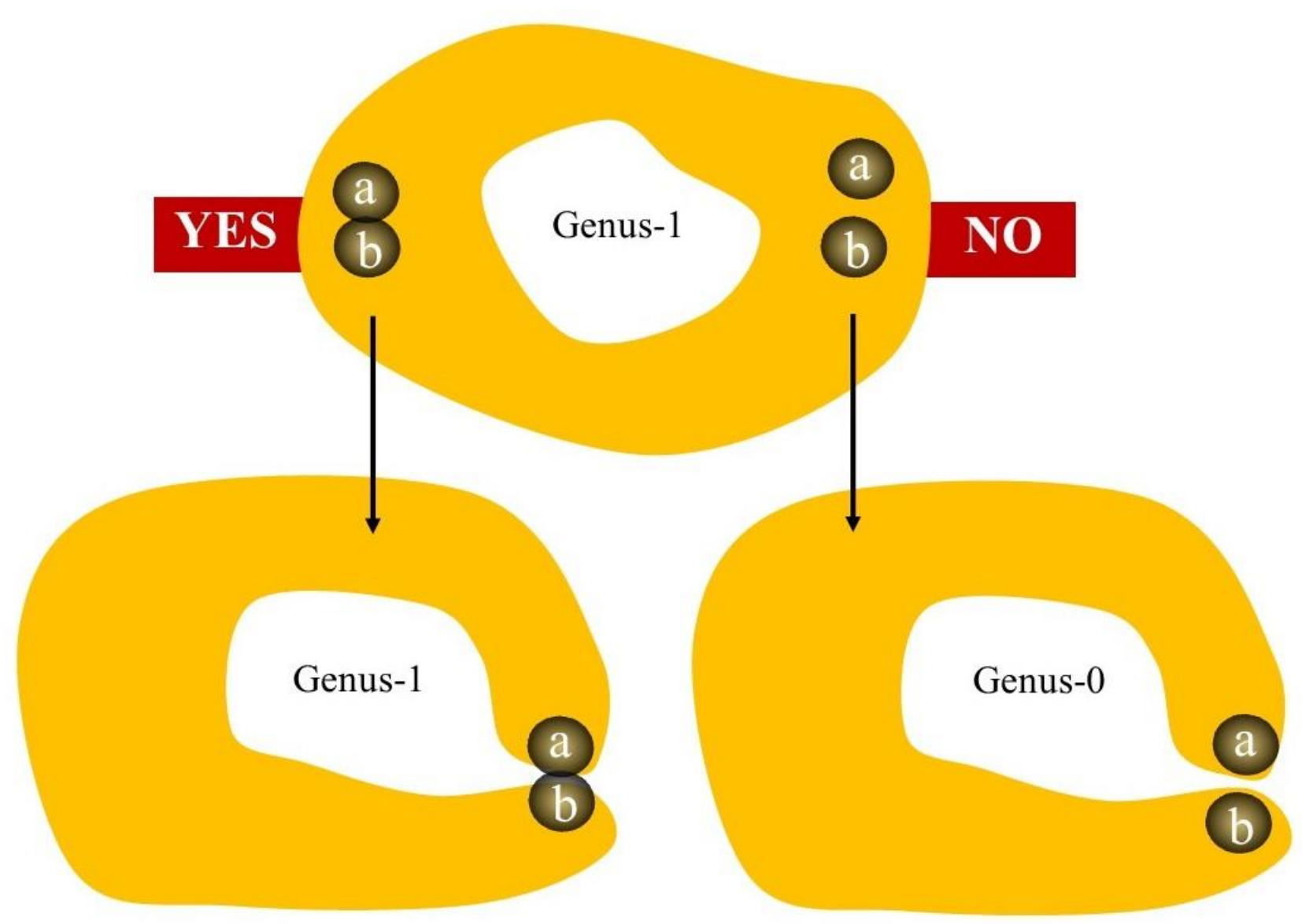

Figure 3. A genus-1 surface cannot contain disjoint points (upper Figure). If two points meet, the hole and the genus are preserved (lower left Figure). In turn, if two points do not meet, the hole gets lost and the surface turns out to be a genus-0 manifold (lower right Figure). 


\section{CONCLUSIONS}

We proposed a negative curvature-based mathematical approach that allows to merge in a single group theoretic framework the operations of both the macroscopic relativistic world and the microscopic quantum realm. It should be remembered that several approaches have already described QM in terms of hyperbolic manifolds. For example, the quantum free particle on the hyperbolic plane can be investigated through the quantization of the Noether momenta if the curvature $\kappa$ is considered as a parameter (Cariñena et al., 2011). Axenides et al. (2021) described the construction of the space of states, of the evolution operators and the continuum limit of the dynamics of a quantum particle, whose phase space is the single-sheeted hyperboloid. Iomin (2013) studied quantum dynamics of a particle in the vicinity of a hyperbolic point, while Khrennikov (2003) developed a quantization formalism in a hyperbolic Hilbert space. The latter Author found that that unitary transformations in hyperbolic Hilbert space represent a new class of transformations of probabilities able to describe a peculiar type of hyperbolic interference. The hyperbolic quantum formalism has bee also used as a new theory of probability waves developed in parallel with the standard QM, since its distinctive feature consists of the restricted validity of the superposition principle (Khrennikov, 2003). This approach leads naturally to covariant thermodynamic fluctuation theory correlated with Riemannian thermodynamic curvature in information geometry (Ruppeiner 1995). The classical and quantum formulations for open systems related to dissipative dynamics are constructed on a complex hyperbolic ring following universal symmetry principles and considering the double thermal fields approach for modeling the system under evaluation and the surrounding environment (Cartas-Fuentevilla et al., 2020). By an experimental standpoint, a step towards on-chip quantum simulation of materials science and interacting particles has been recently achieved in hyperbolic curved space. Indeed, Kollár et al. (2019) used cavity quantum electrodynamics with superconducting circuits and lattices with constant negative curvature as a promising platform for quantum computations/simulations.

Contrary to the above-mentioned studies, we take it a step further. While these Authors claim that QM may be methodologically assessed through a hyperbolic approach, we claim that, as GR really takes place on Euclidean manifolds, QM might really take place on non-Euclidean hyperbolic manifolds. We propose a macroscopic Euclidean world encompassing microscopic regions where a negative-curvature fabric encompasses the paths and waves described by QM. Hyperbolic manifolds are not anymore a methodological tool to approach QM, rather they become part of the description of QM. Hyperbolic manifolds provide a purely mathematical framework where the unification of the symplectic phase space of the macroscopic relativistic world and the noncommutative phase space of the microscopic quantum world might take place.

Another possibility, not explored here, is to use of an involutive functor between the opposite categories of commutativity and noncommutativity, to attain a dagger structure with the adjoint of a morphism being the inverse. This treatment would make it possible to achieve morphisms that are unitary in finite dimensional Hilbert spaces, to build a dagger symmetric monoidal category (Blute and Panangaden, 2011) which is also compact closed, resulting in completeness results that extend the use of Hilbert spaces to relativity issues. To accomplish a dagger category, a fusion category is equipped with a dagger $\dagger$ so that morphisms $f: A \rightarrow B$ have a two-side inverse (Huenen and Karvoven, 2016).

Based on the novel PB theorem, we concluded that the hyperbolic QM manifold must be of genus $\geq 1$ before the measurement. In touch with this observation, it must be emphasized that several scholars before now provided an effort to study quantum waves in genus one manifolds. For example, Biswas and Ghosh (2019) studied the quantum dynamics of point particles moving on non-trivial torus knot, while Narnhofer (2002) discussed reducible representations of particle on toruses with magnetic field (Narnhofer 2002). Kowalski and Rembieliński (2007) examined the coherent states for the quantum mechanics on a torus and their basic properties, while Athanasiu et al. (1996) studied Finite Quantum Mechanics on tori of arbitrary integer discretization, providing the generators of the "rotation group" as well as their quantum mechanical eigenvalues and eigenstates. Giving these premises and the PBT, other physical conclusions can be drawn. A black hole (Chesler 2019), either hyperbolic or not hyperbolic, cannot contain a singularity, unless the black hole itself displays genus $\geq 1$. If a black hole was a genus- 0 manifold (therefore shrinkable to singularity by the huge amount of mass/energy), its own elements would be intersecting. But it runs counter PBT. Hence, the options for a black hole are two:

1) Either the black hole is genus-0 and singularities are not allowed.

2) Or the black hole is genus $\geq 1$ and its elements can be compressed down to a tiny region surrounding the hole(s). 


\section{DECLARATION}

The undersigned Author transfer all copyright ownership of the manuscript, in the event the work is published.

The undersigned author warrants that the article is original, does not infringe on any copyright or other proprietary right of any third part, is not under consideration by another journal, and has not been previously published.

The Author does not have any known or potential conflict of interest including any financial, personal or other relationships with other people or organizations within three years of beginning the submitted work that could inappropriately influence, or be perceived to influence, their work.

This research did not receive any specific grant from funding agencies in the public, commercial, or not-for-profit sectors.

This research does not contain any studies with human participants or animals performed by the Author.

The Author had full access to all the data in the study and take responsibility for the integrity of the data and the accuracy of the data analysis. The Author performed: study concept and design, acquisition of data, analysis and interpretation of data, drafting of the manuscript, critical revision of the manuscript for important intellectual content, statistical analysis, obtained funding, administrative, technical, and material support, study supervision. 


\section{REFERENCES}

1) Aerts, D.; Aerts, S. 2017. Towards a general operational and realistic framework for Quantum mechanics and Relativity Theory, in A. Elitzur, S. Dolev, N. Kolenda, Eds., Quo Vadis Quantum Mechanics, Springer, 2017.

2) Axenides M, Floratos E, Nicolis S. 2021. The arithmetic geometry of AdS2 and its continuum limit. arXiv:1908.06641.

3) Athanasiu GG, Floratos EG, Nicolis S. 1996. Holomorphic quantization on the torus and finite quantum mechanics. J. Phys. A: Math. Gen. 296737.

4) Batygin K. 2018. Schrödinger evolution of self-gravitating discs. Monthly Notices of the Royal Astronomical Society,475(4): 5070-5084. https://doi.org/10.1093/mnras/sty162.

5) Besnard F, Bizi N. 2017. On the definition of spacetimes in Noncommutative Geometry: Part I. arXiv:1611.07830v3.

6) Biswas D, Ghosh S. 2020. Quantum Mechanics of Particle on a torus knot: Curvature and Torsion Effects. EPL (Europhysics Letters), 132(1).

7) Blute, R., Panangaden, P. 2011. Dagger categories and formal distributions. Lecture Notes in Physics 813, Springer, Heidelberg, 421-436, DOI 10.1007/978-3-642-12821-9_6, MR2767050.

8) Calmet X, Fritz C. 2015. Inflation on a Non-Commutative Space-Time. arXiv:1506.04049v1 [hep-th].

9) Cariñena JF, Rañada MF, Santander M. 2011. The quantum free particle on spherical and hyperbolic spaces: A curvature dependent approach. Journal of Mathematical Physics 52, 072104 (2011); https://doi.org/10.1063/1.3610674

10) Cartas-Fuentevilla R, Berra-Montiel J, Meza-Aldama O. 2020. Hyperbolic ring based formulation for thermo field dynamics, quantum dissipation, entanglement, and holography. The European Physical Journal C; 80(603).

11) Cassinelli G, Lahti P. 2017. Quantum mechanics: why complex Hilbert space? Philos Trans A Math Phys Eng Sci. 13;375(2106):20160393. doi: 10.1098/rsta.2016.0393.

12) Chesler PM. 2019. Singularities in rotating black holes coupled to a massless scalar field. arXiv:1905.04613.

13) Comte C. 1996. Symmetry, relativity and quantum mechanics, Il Nuovo Cimento, vol. 111 B, no. 8, $937-956$.

14) Connes, A. 1994. Noncommutative Geometry. Academic Press Inc., San Diego, CA.

15) Cormen TH, Leiserson CE, Rivest RL, Stein C. 2001. Data structures for Disjoint Sets. In: Introduction to Algorithms, MIT Press, 498-524. ISBN 0-262-03293-7.

16) Curtright, T. L.; Fairlie, D. B.; Zachos, C. K. 2014. A Concise Treatise on Quantum Mechanics in Phase Space. World Scientific. ISBN 9789814520430.

17) Das S, Ghosh S, Mignemi S. 2011. Non-commutative spacetime in very special relativity. Physics Letters A. Volume 375, Issue 37, 29 August 2011, Pages 3237-3242. https://doi.org/10.1016/j.physleta.2011.07.024.

18) Dirac PAM. 1925. The Fundamental Equations of Quantum Mechanics. Proceedings of the Royal Society A: Mathematical, Physical and Engineering Sciences. 109 (752): doi:10.1098/rspa.1925.0150.

19) Don AP, Peters JF, Ramanna S, Tozzi A. 2020. Topological View of Flows inside the BOLD Spontaneous Activity of the Human Brain. Front. Comput. Neurosci. DOI: 10.3389/fncom.2020.00034.

20) Eisenberg M, Guy R. 1979. A Proof of the Hairy Ball Theorem. The American Mathematical Monthly, 86 (7): 571-574. doi:10.2307/2320587.

21) Elitzur, A.; Dolev, S.; N. Kolenda, Eds. 2017. Quo Vadis Quantum Mechanics, Springer.

22) Fefferman C, Ivanov S, Kurylev Y, Lassas M, Narayanan H. 2015. Reconstruction and interpolation of manifolds I: The geometric Whitney problem. arXiv:1508.00674

23) Fré, P.G. 2013. Gravity, a Geometrical Course. Vol. 1: Development of the Theory and Basic Physical Applications, Springer, DOI 10.1007/978-94-007-5361-7

24) Ghose, P. 1997. Violatioj of signal locality and unitarity in a merger of quantum mechanics and general relativity, Pramana 49, no. 1, 65-69.

25) Girelli F, Livine ER. 2004. Special relativity as a non commutative geometry: Lessons for deformed special relativity. Phys.Rev. D81 (2010) 085041. DOI: 10.1103/PhysRevD.81.085041

26) Gisin N, Fröwis F. 2018. From quantum foundations to applications and back. Philos Trans A Math Phys Eng Sci. 13;376(2123):20170326. Doi: 10.1098/rsta.2017.0326.

27) Goodman F. 2003. Algebra: Abstract and Concrete, Stressing Symmetry, 2e. Prentice Hall. ISBN 0-13-0673420.

28) Henriques, A. and Penneys, D. 2016. Bicommutant categories from fusion categories. arXiv 1511.05226v2,135.

29) Heuen, C., Karvoven, M. 2016. Monads on dagger categories. Theory and Applications of Categories, 31, no. 35, 1016-1043, MR3584697.

30) Iomin A. 2013. Exponential spreading and singular behavior of quantum dynamics near hyperbolic points. Phys. Rev. E 87, 054901. DOI:https://doi.org/10.1103/PhysRevE.87.054901.

31) Kalau W, Malze M. 1995. Gravity, non-commutative geometry and the Wodzicki residue. Journal of Geometry and Physics, Volume 16, Issue 4, July 1995, Pages 327-344. https://doi.org/10.1016/0393-0440(94)00032

32) Kalckar J, Bohr N, Rosenfeld L, Rüdinger E, Aaserud F. 1996. Foundations of Quantum Physics II (19331958). Elsevier. p. 210. ISBN 978-0-444-89892-0. Retrieved 2011-10-24. 
33) Khrennikov A. 2003. Hyperbolic quantum mechanics. Advances in Applied Clifford Algebras, 13:1-9.

34) Kollár AJ, Fitzpatrick M, Houck AA. 2019. Hyperbolic lattices in circuit quantum electrodynamics. Nature, 571: 45-50.

35) Kowalski K, Rembieliński J. 2007. Coherent states for the quantum mechanics on a torus. Phys. Rev. A 75, 052102.

36) Matoušek J. 2003. Using the Borsuk-Ulam Theorem. Lectures on Topological Methods in Combinatorics and Geometry. Berlin Heidelberg: Springer-Verlag.

37) Narnhofer H. 2002. Quantum theory on the torus with magnetic field. Quantum Theory and Symmetries, 144159. https://doi.org/10.1142/9789812777850_0013.

38) Padavić K, Sun K, Lannert K, and Vishveshwara S. 2020. Vortex-antivortex physics in shell-shaped BoseEinstein condensates. Phys. Rev. A 102, 043305.

39) Pesin YB .1981. Geodesic flows with hyperbolic behaviour of the trajectories and objects connected with them. Russian Mathematical Surveys, 36 (4).

40) Peters JF. 2016. Computational Proximity. Excursions in the Topology of Digital Images. Intelligent Systems Reference Library 102. Switzerland: Springer Int. Pub. DOI: 10.1007/978-3-319-30262-1.

41) Piazza L, Lummen TT, Quiñonez E, Murooka Y, Reed BW, Barwick B, Carbone F. 2015. Simultaneous observation of the quantization and the interference pattern of a plasmonic near-field. Nat Commun. 2015 Mar 2;6:6407. doi: 10.1038/ncomms7407.

42) Po HC, Watanabe H, Vishwanath A. 2018. Fragile Topology and Wannier Obstructions. Phys. Rev. Lett. 121, 126402.

43) Ruppeiner G. 1995. Riemannian geometry in thermodynamic fluctuation theory. Rev. Mod. Phys. 67, 605.

44) Schroers BJ. 2011. Quantum gravity and non-commutative space-times in three dimensions: a unified approach. Acta Physica Polonica B Proceedings. Vol. 4 (2011) Supplement No 3

45) Sengupta B, Tozzi A, Coray GK, Douglas PK, Friston KJ. 2016. Towards a Neuronal Gauge Theory. PLOS Biology 14 (3): e1002400. doi:10.1371/journal.pbio.1002400.

46) Skalsky, V. 2010. The model of a flat (Euclidean) expansive homogeneous and isotropic relativistic universe in the light of the general relativity, quantum mechanics, and observations. Astrophys. Space Sci. 330, 373-398, DOI 10.1007/s10509-010-0409-8

47) Tegmark M. 2008. The Mathematical Universe. Foundations of Physics. February 2008, Volume 38, Issue 2, pp 101-150.

48) Tozzi A, Peters JF, Fingelkurts AA, Fingelkurts AA, Marijuán PC. 2017. Topodynamics of metastable brains. Physics of Life Reviews, 21, 1-20. http://dx.doi.org/10.1016/j.plrev.2017.03.001

49) Tumulka, R. 2009. Orthonormal basis. In: D. Greenberger, K. Hentschel, F. Weinert, eds., Compendium of Quantum Physics, Springer, Berlin, https://doi-org.uml.idm.oclc.org/10.1007/978-3-540-70626-7_135.

50) Ungar AA. 2005. Analytic Hyperbolic Geometry: Mathematical Foundations and Applications. World Scientific, ISBN: 978-981-256-457-3.

51) Ungar AA. 2013. Gyrogroups, the Grouplike Loops in the Service of Hyperbolic Geometry and Einstein's Special Theory of Relativity. arXiv:1303.0218.

52) Yilmaz, H. 1981. Relativity and quantum mechanics, Int. J. of Theoretical Physics 21, no. 10/11, 871-902. 